\title{
Estudio de estabilizadores en el adobe
}

\section{Study of stabilizeris in adobe}

\section{Paredes Avilés Freddy Leoncio}

Ingeniero Civil; Especialista en Gestión de Proyectos; Magister en Docencia Universitaria; Docente en el Instituto Metropolitano de Diseño; Docente en la Universidad Central del Ecuador

flparedes@uce.edu.ec

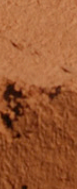

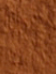

\section{a} De la Cruz Arce Gloria Magdalena

Arquitecta; Magister en Docencia Universitaria; Docente en el Instituto Metropolitano de Diseño; Docente en la Universidad Central del Ecuador

gmdelacruz@uce.edu.ec

\section{Resumen}

Se elaboraron de adobes con dos tipos de tierra llamadas cangahua y arcilla con la inclusión de diversos materiales aglomerantes. El objetivo es mejorar su resistencia a la compresión, utilizando aglomerantes naturales y artificiales. Esta investigación permitió contrastar su importancia en el desarrollo de la vivienda en muchos lugares del mundo a través de la historia y que lamentablemente ha sido desplazado, como elemento constructivo, por el aparecimiento de otros materiales.

Los aglomerantes utilizados son materiales naturales como: paja de páramo, cáscara de arroz, fibra de cáscara de coco y un elemento que no es natural, el catalizador gastado del proceso de refinamiento de combustibles compuesto de sílice y aluminio, en dosificaciones del 5, 10, 15 y $20 \%$ tanto con cangagua, como con arcilla, lo que determinó la nueva resistencia de los adobes.

Las muestras elaboradas, se ensayaron en el laboratorio de la Facultad de Ingeniería Ciencias Físicas y Matemáticas de la Universidad Central del Ecuador, dónde fueron sometidas a la resistencia a la compresión, logrando obtener resultados comparables con las normas mínimas de resistencia que el INEN exige para materiales que cumplen iguales funciones en la construcción. El comparativo es con el bloque hueco de cemento y el ladrillo cerámico.

Los resultados obtenidos evidencian que: la muestra de cangahua en un $80 \%$ más el $20 \%$ de fibra de cáscara de coco (4,9MPa) sobrepasa la resistencia del bloque hueco tipo B (4MPa) y la resistencia del ladrillo tipo E (4 MPa).

Palabras claves: sustentabilidad; tecnología; resistencia; dosificación; catalizador; compresión

Abstract: It has been made adobes with two kinds of soil, Cangahua and clay with the inclusion of different binder materials. The objective is to improve the compression strength of the material, using natural and artificial binders. This research allowed to verify its importance in history of housing development in many places around the world. Regrettably, it has been replaced as a constructive material because of new materials. Some binders used are natural materials like: mountain straw, rice husk, coconut shell fiber.

Another non-natural element used is the remaining material of the fuel refining process which is compound by silica and aluminium. It was used in 5, 10, 15,20\% dosages with canguagua and with clay. It determined a new adobes's resistance. The samples was tested in laboratories of the Faculty of physical science and mathematics engineering at Universidad Central del Ecuador. They were subjected to compressive loads where the results were comparable with the minimum material requirement that standard INEN specify in constructions. The comparative is with hollow cement blocks and ceramic brick.

The results show that: Cancagua sample $80 \%$ plus 20\% of coconut shell fiber (4.9MPa), surpasses the resistance of the hollow cement block type B (4MPa) and brick type E (4MPa). 


\section{Introducción}

A lo largo del país y especialmente en la sierra se observa que el adobe juega un papel importante en la construcción, no solo en áreas rurales donde es utilizado por los pobladores de la zona, sino también en áreas urbanas, sobreviviendo a través de los siglos, resistiendo el embate de sismos y terremotos, evidencia que se encuentra, sobre todo en los centros históricos.

El adobe es un aislante térmico que propicia un ambiente confortable para el ser humano. Tiene la virtud de eliminar la electrostática que se acumula en las personas con tan solo tocar la superficie de las paredes, lo que nos ha llevado a tomar como tema de estudio el adobe y su comportamiento, con la inserción de nuevos aglomerantes.

Dentro del proceso de estudio se tomó muestras de tierra de diferentes lugares, tanto en la ciudad de Quito como en Echeandía, provincia de Bolívar, las mismas que sometimos a los respectivos análisis, para saber si estas cumplen con los requisitos para fabricar adobes.

Se trabajó con materiales aglomerantes como: cáscara de arroz, fibra de cáscara de coco, paja de páramo y el catalizador FCC. Con los materiales antes expuestos se elaboró los adobes en forma artesanal.

Una vez secos los adobes, fueron trasladados al laboratorio de ensayo de materiales de la Facultad de Ingeniería Ciencias Físicas y Matemáticas, de la Universidad Central del Ecuador donde se realizó la prueba a la compresión para determinar su resistencia.

\section{Método}

\section{Diseño del estudio}

\section{a. Definición de Variables:}

Variable independiente: Adobes de cangagua y arcilla. Variable dependiente: Resistencia a la compresión.

b. Medición de Variables y Procedimientos:

\section{Procesos}

\section{Proceso uno}

\section{Sujetos y tamaños de muestra}

Se tomaron 10 muestras en diferentes lugares de la ciudad de Quito y en Echeandía, de los cuales se definieron dos que presentaban las características propicias para la elaboración de adobes.

\section{Toma de muestras}

La toma de las muestras se efectuó en la Calle Francisco Barros OE7-135 y Carrascal del Barrio Pichincha de la ciudad de Quito, zona donde antiguamente se elaboraba adobes, ladrillos y tejas; así como, en la Provincia de Bolívar en el Centro Turístico Altamira de Echeandía.

\section{MUESTRA UNO}

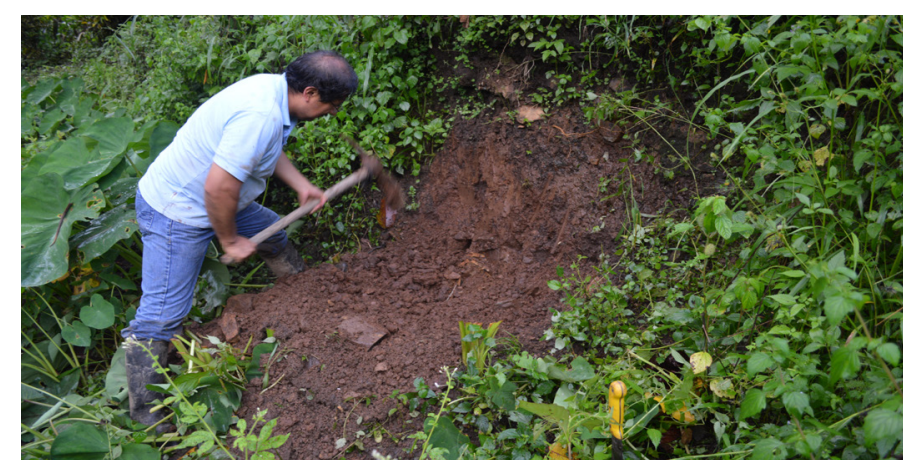

Foto1.- Toma de muestras de arcilla, en el Centro Ecoturístico Altamira de Echeandía, provincia de Bolívar, fuente: Arq. Gloria De La Cruz Arce

\section{MUESTRA DOS}

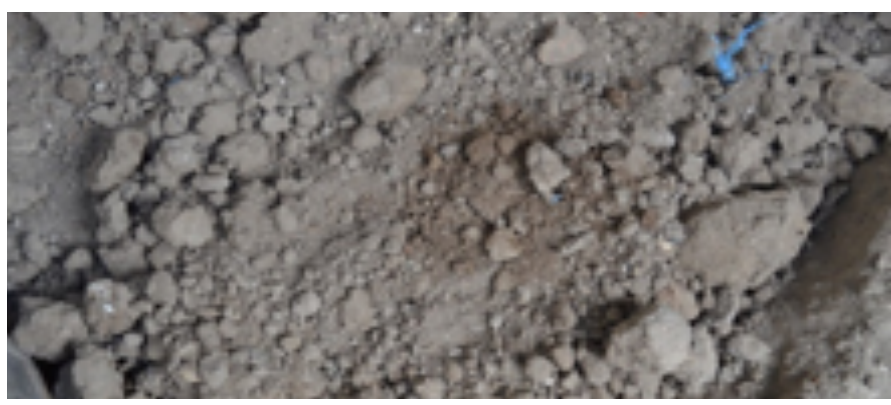

Foto 2.- Muestras de tierra tomada en la calle Francisco Barros OE7-135 y Carrascal, Barrio Pichincha-Quito, fuente: Ing. Freddy Paredes Avilés.

\section{Proceso dos: Elaboración de los Adobes}

El proceso de elaboración de los adobes parte desde el análisis de la tierra seleccionada en la ciudad de Quito. El estudio de sueldos determinó que la muestra tiene: $4,82 \%$ de grava, $51,4 \%$ de arena y $43,94 \%$ de finos; un límite líquido de $38,10 \%$, un límite plástico de $29,99 \%$, un índice plástico del $8,11 \%$ dándonos como resultado un suelo tipo ML Limo de baja plasticidad.

El uso del catalizador de Craqueo Catalítico Fluidizado (FCC) utilizado en la Refinería Esmeraldas, que está compuesto de base silicoaluminosa y estructura zeolítica cuando pierde sus propiedades catalíticas, se debe reemplazar por uno nuevo, el residuo de catalizador desactivado se desecha y se almacena como un residuo industrial. En la realización de hormigones ha dado un mejoramiento en su resistencia de un $10 \%$.

El estudio realizado en la muestra de la ciudad de Quito, evidenció un alto porcentaje de grava. La tierra fue tamizada para poder eliminar los agregados gruesos. 
Al mismo tiempo, se procede a preparar los aglomerantes: la paja de páramo se corta en tamaños aproximados de $10 \mathrm{~cm}$., la fibra de la cáscara de coco se desmenuza finamente y luego se corta en tamaños aproximados de $10 \mathrm{~cm}$., la cáscara de arroz, al ser de tamaño pequeño, no requiere preparación alguna; mientras que, el catalizador FCC se coloca en proporción de 5, 10, 15 y 20\% con relación a la tierra.

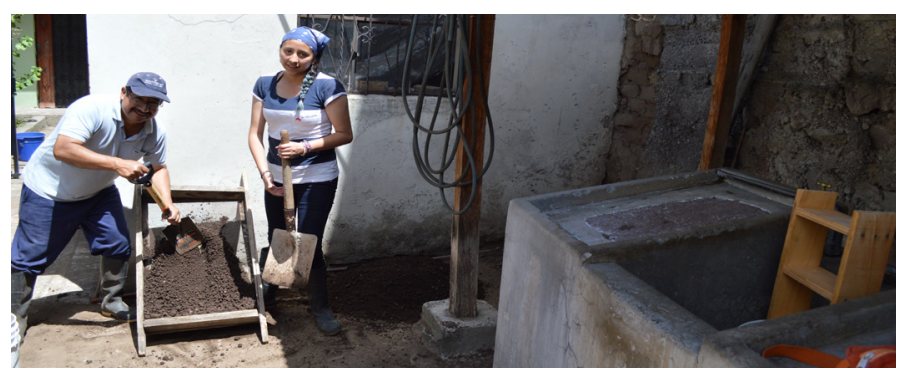

Foto 3.- Tamizado de la tierra, fuente: Arq. Gloria De La Cruz Arce

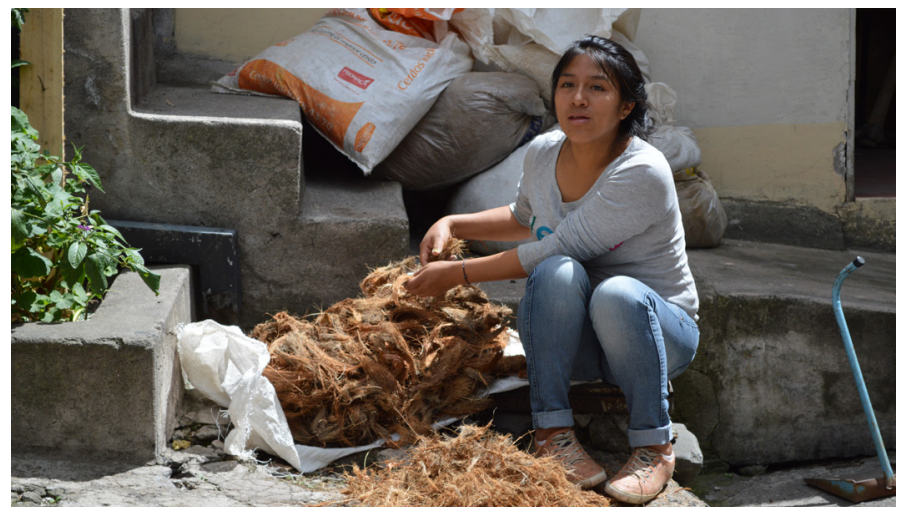

Foto 4.- Preparando la fibra de coco, fuente: Ing. Freddy Paredes Avilés.

Se fabricó una adobera con las siguientes dimensiones: largo $20 \mathrm{~cm}$, ancho $15 \mathrm{~cm}$, altura $10 \mathrm{~cm}$., la misma que se dejó por 12 horas en remojo con abundante agua, para que absorba la suficiente humedad y evitar que los adobes se peguen al molde.

\section{Proceso de fabricación de adobe con fibra de coco}

Como se puede observar en la foto, se utilizó como aglomerante la fibra de coco en dosificaciones del $5 \%, 10 \%, 15 \%$ y $20 \%$. Es preciso anotar que las mismas cantidades se utilizaron con otros aglomerantes. Se trabajó con una muestra de 5 adobes por cada por-centaje.

Cabe señalar que tanto la fibra de coco como el catali-zador FCC, fueron utilizados por primera vez en esta investigación, ya que no se logró evidenciar trabajos anteriores con estos materiales.

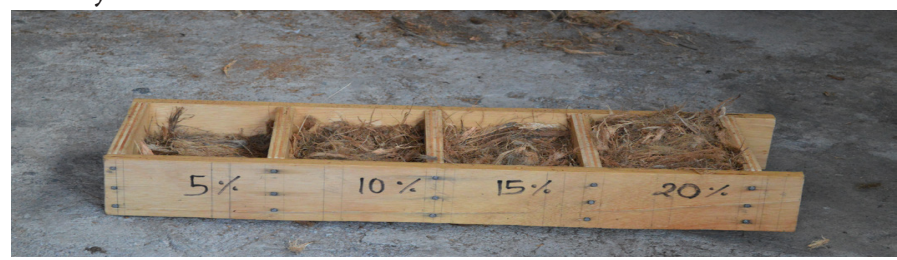

Foto 5.- Adobera con las dosificaciones del 5\%, 10\%, 15\%, $20 \%$ de fibra de coco, fuente: Ing. Freddy Paredes Avilés.

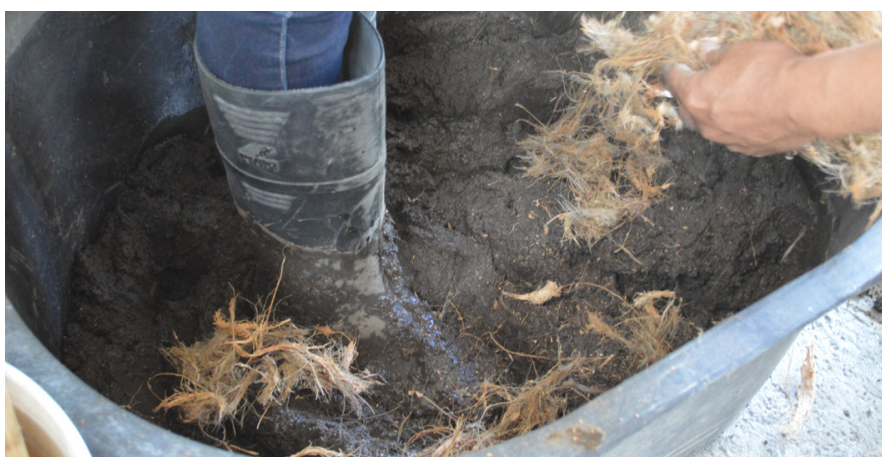

Foto 6.- Mezcla de la tierra húmeda (chocoto) con la fibra de coco, fuente: Ing. Freddy Paredes Avilés.

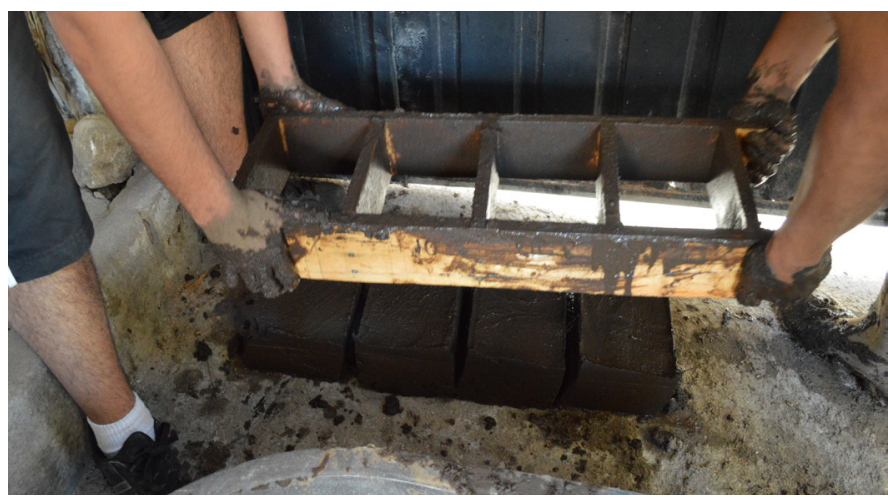

Foto 7.- Elaboración de los adobes, fuente: Arq. Gloria De La Cruz Arce.

Una vez terminados los adobes, se procedió al etiquetado para diferenciar en cada una las dosificaciones de los materiales estabilizadores y hacer el seguimiento correspondiente.

Este proceso se repitió para la paja de páramo, la cáscara de arroz y el catalizador FCC.

A las veinte y cuatro horas de terminado el proceso, se observó que los adobes que contenían fibras cortas como la cáscara de arroz o el catalizador sufrían fisuras de ancho considerable.

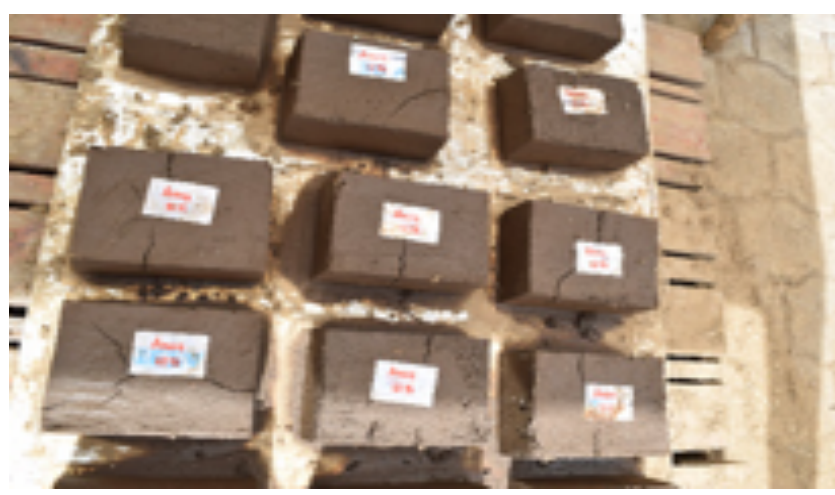

Foto 8.- Adobes con cáscara de arroz, donde se evidencia las fisuras en su superficie, fuente: Ing. Freddy Paredes Avilés.

A los cuatro días de elaborados, se procedió a voltearlos y este procedimiento se realizó periódicamente por un lapso de 30 días. Una vez cumplido este tiempo, fueron llevados al laboratorio de Ensayo de Materiales de la Facultad de Ingeniería, donde se los alisó, pesó y midió, para 
luego someterlos a la compresión.

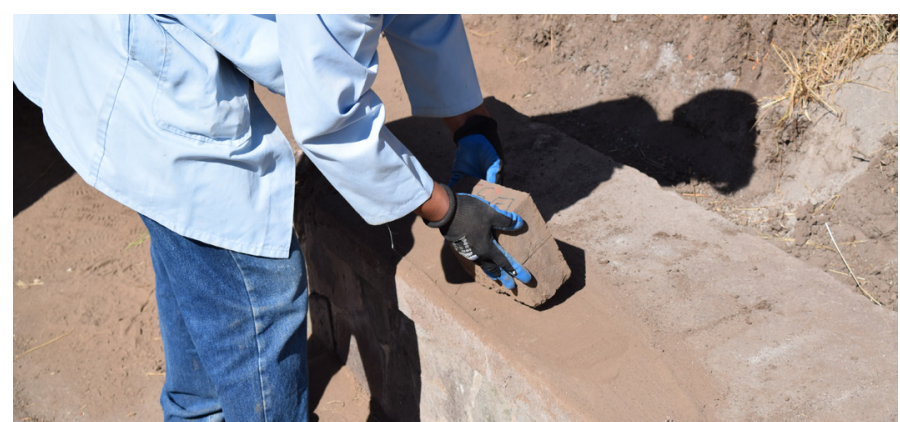

Foto 9.- Preparación de los adobes para ser sometidos a la compresión, fuente: Ing. Freddy Paredes Avilés.

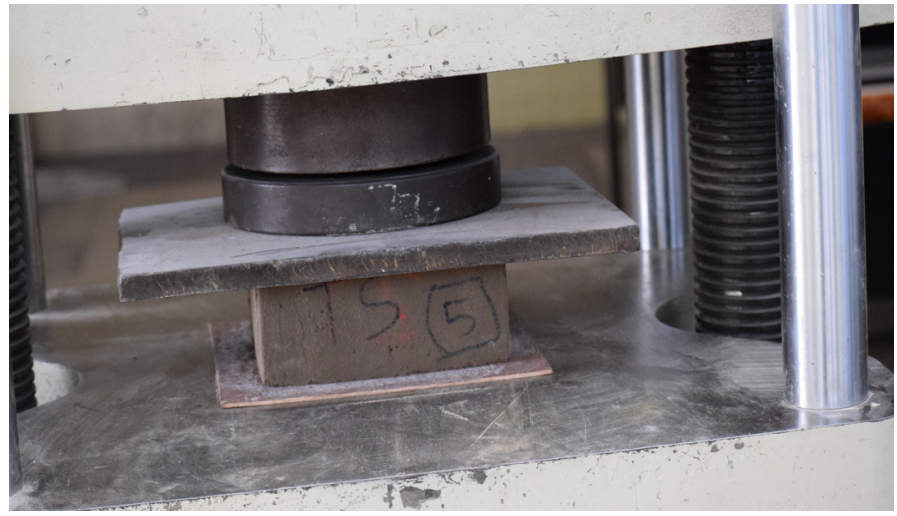

Foto 10.- Adobes sometidos a la compresión, fuente: Ing. Freddy Paredes Avilés.

\section{Resultados}

Con los datos obtenidos al esfuerzo de compresión, se procedió a sistematizarlos, luego fueron promediados, obteniendo los resultados que exponemos a continuación:

Tabla 1. Relación tierra - Cáscara de arroz

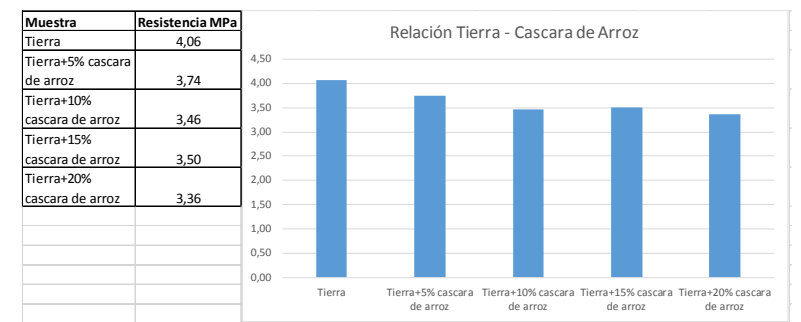

Elaborado por: Ing. Freddy Paredes Avilés / Fuente: Ing. Freddy Paredes Avilés Arq. Gloria De La Cruz Arce

Tabla 2. Relación tierra - Paja de páramo

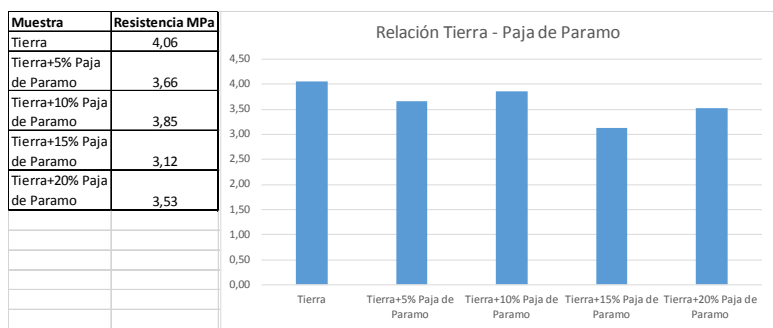

Elaborado por: Ing. Freddy Paredes Avilés / Fuente: Ing. Freddy Paredes Avilés Arq. Gloria De La Cruz Arce
Tabla 3. Relación tierra - fibra de coco

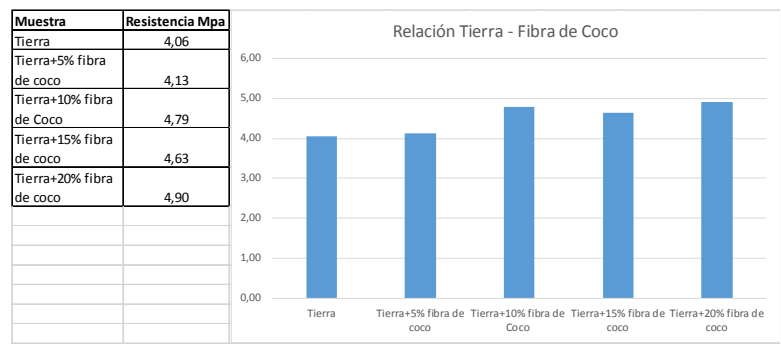

Elaborado por: Ing. Freddy Paredes Avilés / Fuente: Ing. Freddy Paredes Avilés - Arq. Gloria De La Cruz Arce

Tabla 4. Relación tierra - 20 \% fibra de coco - catalizador gastado

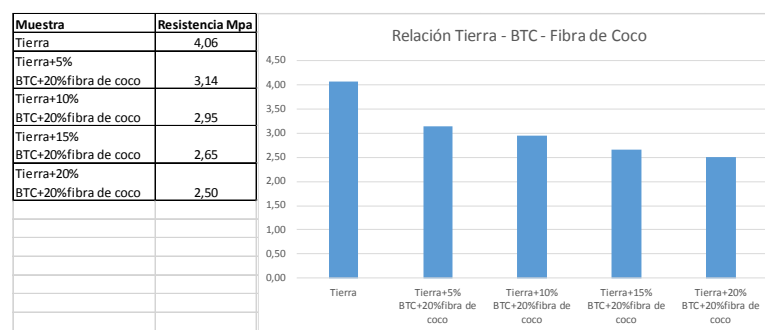

Elaborado por: Ing. Freddy Paredes Avilés / Fuente: Ing. Freddy Paredes Avilés Arq. Gloria De La Cruz Arce

\section{Discusión}

En las construcciones actuales se utiliza bloques huecos de hormigón simple en un alto porcentaje y ladrillos cerámicos en menor proporción, para mampostería.

El INEN ha establecido como Norma Técnica Ecuatoriana Obligatoria para los bloques huecos de hormigón los siguientes requisitos:

\begin{tabular}{lc}
\hline TIPO & $\begin{array}{l}\text { Resistencia mínima a la } \\
\text { compresión en MPa a los }\end{array}$ \\
BE & 28 días (Norma INEN 640) \\
\hline A & 6 \\
B & 4 \\
C & 3 \\
D & 2,5 \\
E & 2
\end{tabular}

De igual manera, el INEN ha establecido como Norma Técnica Ecuatoriana Obligatoria para los ladrillos cerámicos empleados en la construcción los siguientes requisitos:

\begin{tabular}{lc}
\hline TIPO & $\begin{array}{l}\text { Resistencia mínima a la } \\
\text { compresión en MPa }\end{array}$ \\
DE & Individual (INEN 297) \\
LADRULLO & 20 \\
Macizo tipo A & 14 \\
Macizo tipo B & 6 \\
Macizo tipo C & 5 \\
Hueco tipo D & 4 \\
Hueco tipo E & 3 \\
Hueco tipo F &
\end{tabular}


Si se compara la resistencia a la compresión exigida por el INEN - 640 para los bloques huecos de hormigón con los resultados obtenidos de las muestras de adobe con cáscara de arroz, paja de páramo, fibra de coco, tierra sola y el catalizador (cuadro de resultados) se concluye que:

a. Ninguna de las muestras sobrepasa la resistencia del bloque tipo A.

b. Las muestras de fibra de coco y la muestra de tierra sola sobrepasan la resistencia del bloque tipo B.

c. Las muestras con cáscara de arroz, con paja de páramo y con el catalizador sobrepasan la resistencia de los bloques tipo C.

Si comparamos la resistencia a la compresión exigida por el INEN - 297 para los ladrillos cerámicos con los resultados obtenidos de las muestras de adobe elaboradas con cáscara de arroz, paja de páramo, fibra de coco, tierra sola y el catalizador (cuadro de resultados) se concluye que:

a. Ninguna de las muestras sobrepasa la resistencia de los ladrillos macizos tipo A, B y C, y del ladrillo hueco tipo D.

a. Las muestras de fibra de coco y la muestra de tierra sola sobrepasan la resistencia del ladrillo hueco tipo E.

a. Las muestras con cáscara de arroz, con paja de páramo y con el catalizador sobrepasan la resistencia del ladrillo hueco tipo F.

\section{Conclusiones}

\section{Adobes con tierra sola}

Es importante señalar que estos adobes presentan pequeñas fisuras superficiales producto del proceso de secado, por lo que es recomendable que se coloque aglomerantes en su composición, para evitar el agrietamiento de los mismos y obtener mejor resistencia a la compresión.

Sometidos a una carga uniforme sobre el área a ensayar a la compresión, ésta se fisura y se rompe.

Los resultados de estos adobes, se toman como muestras comparativas en relación con los adobes elaborados con aglomerantes, donde se supone que, éstos últimos mejoraran el comportamiento a la resistencia o el efecto destructivo.

\section{Compresión con cáscara de arroz}

Como se puede visualizar (foto 8) se producen fisuras en los adobes, debido a que la cáscara de arroz es muy pequeña, lo que no permite una mayor cohesión de la tierra

El resultado a la compresión, observando la tabla 1, relación tierra - cáscara de arroz, se deduce que, al incrementar el porcentaje de aglomerante, el adobe baja su resistencia, a tal grado que es inferior al del adobe de tierra sola.

En consecuencia, de acuerdo a los datos obtenidos, esta mezcla no es remendable.

\section{Compresión con paja de páramo}

Los resultados obtenidos en el ensayo relación tierra - paja de páramo en la tabla 2, se observa que a mayor dosis de aglomerante se produce menor resistencia. La pregunta es ¿por qué históricamente se utiliza esta fibra natural para hacer los adobes?

La respuesta está en que, al observar tanto en las construcciones antiguas, como en los adobes realizados para este estudio, éstos presentan muy pocas fisuras, es decir, se sacrifica un poco la resistencia para ganar la cohesión de la tierra, evitando que los adobes se rompan en el proceso de secado, traslado y ubicación en obra.

\section{Compresión con fibra de coco}

La relación tierra - fibra de coco en la tabla 3, mejora notablemente la resistencia del adobe conforme se aumenta el aglomerante, con lo que podemos deducir que, a mayor cantidad de fibra de coco, mayor es la resistencia a la compresión.

Esta mixtura presenta una pequeña desventaja, a mayor cantidad de fibra, el proceso de mezclado con la tierra húmeda se dificulta.

Con fibra de coco se pudo observar que se obtienen dos comportamientos:

b. Resistencia a la compresión: cuando el adobe falla sometido a la carga de compresión.

c. Resistencia a la compactación o aplastamiento: luego de la falla visible a la carga de compresión, el adobe sigue ganando resistencia, porque la fibra de cáscara de coco, actúa como una malla que evita que se desintegre la tierra, manteniendo su resistencia por mayor tiempo.

\section{Compresión con fibra de coco al $20 \%$ y catalizador FCC}

En la tabla 4, relación tierra - $20 \%$ fibra de coco - catalizador FCC, se observa que, de la resistencia ganada por la fibra de coco, ésta baja dramáticamente con el aglomerante FCC, en todos los porcentajes que fueron agregados. 
En consecuencia, esta mezcla no es factible reproducirla, la resistencia del adobe baja en un $40 \%$.

Las muestras elaboradas con fibra de coco y con tierra sola, arrojaron mejores resultados en los ensayos a la compresión.

Los ensayos a la compresión realizados en las muestras elaboradas con cáscara de arroz y con catalizador, dieron resultados no aptos para la elaboración de adobes.

Con las muestras de: cáscara de arroz, paja de páramo, fibra de coco, tierra sola y el catalizador, se pudo obtener mayores resultados a la compresión que la Norma INEN 640 establece para los bloques B, C, D y E.

Con las muestras de cáscara de arroz, paja de páramo, fibra de coco, tierra sola y el catalizador, se pudo obtener mayores resultados a la compresión que la Norma INEN 297 establece para los ladrillos cerámicos huecos tipo $\mathrm{E}$ y $\mathrm{F}$.

En consecuencia, se concluye que, de los resultados obtenidos, se puede continuar efectuando investigaciones más profundas con otros aglomerantes y a su vez relegar el proceso artesanal para desarrollar modelos semi industriales, que sean fáciles de adoptar para mejorar la resistencia del adobe.

De igual manera trabajar con diversos tipos de tierras, de tal manera que podamos tener un comportamiento promedio por zona o por regiones del Ecuador.

\section{Referencias Bibliográficas}

- Norma INEN 0297 (1978): Ladrillos cerámicos. Requisitos

- Norma INEN 0640 (1993): Bloques huecos de hormigón. Requisitos

- Gernot Minke, 2005, Manual de Construcción en Tierra, Alemania, Editorial Fin de Siglo.

- Blondet, M., Torrelva, D. and Villa García, G. (2002). Adobe in Peru: Tradition, Research and future. Modern Earth Building 2002 - International Conference and fair, Berlin, Germany.

- Shiller Silvia, Martin Evans John, 2007, Construcción con tierra 3, Ciudad de Buenos Aires, Argentina, IAA.

- Morales Morales Roberto, Manual Construcción de Viviendas de Adobe, Ciudad de Lima, Perú, Talleres Gráficos de Victor Castillo.

- Gernot Minke, 2013, Manual de Construcción con tierra, ciudad de Buenos Aires, Argentina, BRC Ediciones.

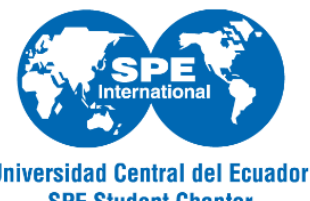

SPE Student Chapter
El SPE Student Chapter Universidad Central del Ecuador, es un programa sin fines de lucro de la SPE Internacional creado para el apoyo académico-profesional de los estudiantes afines a la industria petrolera.

A nivel mundial existen 367 capítulos estudiantiles presentes en 130 países, en el Ecuador 5 de estos vienen contribuyendo al desarrollo de los futuros profesionales del área hidrocarburífera: ESPOL, UTE, UPSE, EPN y UCE.

El SPE Student Chapter UCE fue establecido el primero de junio de 1999, en la actualidad consta de 150 miembros distribuidos en diferentes carreras como: Ingeniería en Geología, Ambiental, Química.

Los representantes estudiantiles se eligen anualmente, la directiva para el período 2016 - 2017 se encuentra conformada por miembros de distintos semestres de la Carrera de Ingeniería de Petróleos:

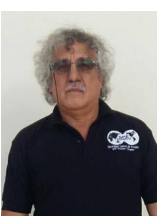

El trabajo de la directiva necesita de una guía para desarrollar su labor siempre en-marcado en las políticas de la sección Ecuador, el Faculty Advisor proporciona un vínculo crítico entre los dos entes, tarea que ha sido llevada con responsabilidad y liderazgo por el Ing. Gustavo Pinto.

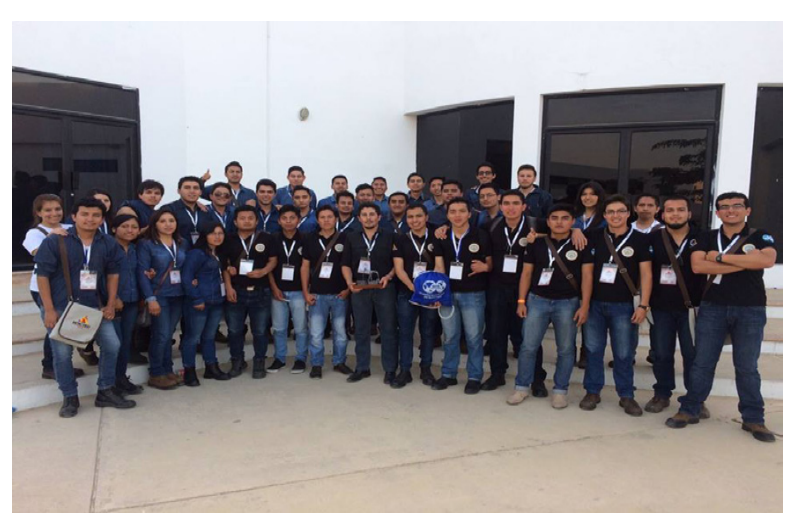

Delegación UCE en el concurso PETROTEST 2016 - UPSE Prov. Santa Elena

mientas que nos otorga la SPE.

- En mayo con el auspicio de la empresa SERTECPET se desarrolló el curso "Diseño y selección del levantamiento artificial hidráulico tipo jet para pozos petroleros" 